\title{
Energy-harvesting materials for smart fabrics and textiles
}

Russel Torah, Jake Lawrie-Ashton, Yi Li, Sasikumar Arumugam, Henry A. Sodano, and Steve Beeby

Russel Torah, Department of Electronics and Computer Science, University of Southampton, UK; rnt@soton.ac.uk

Jake Lawrie-Ashton, Department of Electronics and Computer Science, University of Southampton, UK; Jla1g13@soton.ac.uk

Yi Li, Department of Electronics and Computer Science, University of Southampton, UK; yi.li@soton.ac.uk

Sasikumar Arumugam, Department of Electronics and Computer Science, University of Southampton, UK; S.Arumugam@soton.ac.uk

Henry Sodano, Department of Aerospace Engineering, Department of Materials Science and Engineering, Department of Macromolecular Science and Engineering, University of Michigan, USA; hsodano@umich.edu

Steve Beeby, Department of Electronics and Computer Science, University of Southampton, UK; spb@soton.ac.uk

This article reviews materials developed to enable energy harvesting from textiles. It includes energy harvesting from mechanical, thermal, and light sources and covers materials employed into yarns that can be woven into the textile and films that are deposited onto the surface of the textile. The textile places challenging constraints on the materials, for example by limiting processing temperatures to typically less than $150^{\circ} \mathrm{C}$ and presenting a rough, inconsistent surface profile. Example materials include a screen-printable low-temperature composite lead zirconate titanate polymer film and polyvinylidene fluoride polymer fibers, both of which have been shown to harvest mechanical energy from textiles. Thermoelectric solutions demonstrated thus far are limited and challenging to implement within a textile. Photovoltaic solutions include organic and dye- 
sensitized solar cells fabricated into functionalized yarns and as films spraycoated onto textiles. While numerous suitable example materials and textile devices have been demonstrated, work is still needed to develop these into practical energy-harvesting solutions.

Keywords: Piezoelectric, thermoelectric, photovoltaic

\section{Introduction}

Energy harvesting is the conversion of ambient energy into electrical energy for use in powering autonomous electronic devices or circuits. Energy can be harvested from mechanical sources (e.g., strain, displacements), thermal gradients, or incident light (photovoltaics). While numerous examples of such energy harvesters exist, incorporating the required materials into a textile presents unique challenges, both in terms of fabrication and implementation. This article discusses energy-harvesting materials that can be combined with textiles to enable the textile itself to act as the energy harvester.

Energy-harvesting textiles can be achieved by adding active materials as films on the surface of the textile, or as yarns that are woven or embroidered into the textile. The film approach enables low-cost printing processes to be used. This has the advantages of being familiar to the textile industry and can be used to deposit the material across large areas. It also enables the energy-harvesting ability to be added to a wide range of otherwise standard textiles. The disadvantages of this approach derive from processing challenges and constraints the textile places on the functional materials. Processing temperatures are limited to around $150^{\circ} \mathrm{C}$, and the otherwise rough and "fluffy" surface requires the textile to be planarized with an interface layer. ${ }^{1}$ The printed films change the feel of the textile, and the printed area needs to be minimized to reduce this effect. Active yarns, however, can be fabricated separately and then woven into the textile, typically alongside standard textile yarns. This approach places fewer constraints on functional materials, but their positioning in the textile is limited to the warp and weft directions of the textile. The warp and weft of the fabric relate to the weave structure; yarns in the warp direction are held in tension longitudinally and 
the weft yarns are drawn perpendicular to the warp, weaving over and under the warp yarns. The mechanical properties of the active yarns may also affect the feel of the textile, and they must be sufficiently robust to withstand the weaving, knitting, or embroidery process.

Textile energy harvesting can potentially be used as an alternative to batteries, which contain a finite amount of energy and require periodic replacement or recharging. In wearable textile applications, batteries are rigid bulky items that must be removed before washing. The development of energyharvesting textiles is relatively new and has the potential to power e-textiles directly from the fabric itself.

\section{Materials for mechanical energy harvesting}

Piezoelectric materials are well suited for energy harvesting since their inherent energy transduction capability offers direct energy conversion that is subject to strain without the need for external conditioning or bias voltages. The level of energy transduction in a piezoelectric material is defined by the piezoelectric and voltage coefficient of the material. The piezoelectric coefficient, $d(p C / N)$, correlates the generated charge to the applied stress and the voltage coefficient, $g$ $(\mathrm{Vm} / \mathrm{N})$, relates the generated electric field to the applied stress. The most commonly used piezoelectric materials for energy harvesting are piezoceramics with the perovskite structure, ${ }^{2}$ such as lead zirconate titanate (PZT), which has a maximum piezoelectric coefficient, $d_{33}$, of approximately $600 \mathrm{pC} / \mathrm{N}$ which is a significantly high value for use in energy harvesting. However, lead-based piezoelectric materials were recently exempted from use by the European Union directives regarding the use of toxic materials. Efforts have sought to identify alternative compositions that exhibit high electromechanical coupling. An example of recent developments is a solid solution at the morphotropic phase boundary such as $\mathrm{Ba}\left(\mathrm{Ti}_{0.8} \mathrm{Zr}_{0.2}\right) \mathrm{O}_{3}-\left(\mathrm{Ba}_{0.7} \mathrm{Ca}_{0.3}\right) \mathrm{TiO}_{3}$, which shows a high piezoelectric coefficient of $d_{33} \sim 620 \mathrm{pC} / \mathrm{N}$ at optimal composition. ${ }^{3}$

There are numerous methods for integrating piezoelectric materials into a textile, however, the brittle nature of ceramics leads to their application as a piezoelectric cladding on the surface of an inactive fiber. Sodano et al. developed 
examples of piezoceramic clad fibers using $\mathrm{PZT}^{4,5}$ and barium titanate ${ }^{6}$ on conductive core fibers such that one electrode was internal to the fiber and thus eliminating shorting. An alternative methodology to overcome the low strain to failure is through the use of nanoscale materials, such as vertically aligned $\mathrm{ZnO}$ nanowires that were demonstrated to harvest energy from the sliding contact between two separate fibers yielding high energy extraction. ${ }^{7}$

Malakooti et al. ${ }^{8}$ grew $\mathrm{ZnO}$ nanowires directly onto Kevlar fabrics and used these for energy harvesting (Figure 1). Rather than using a thin film on a fiber core, the electrospinning process allows the direct manufacture of nonwoven fabrics with sufficiently small diameters to enable a high degree of flexibility. This has also been applied to the formation of piezoceramic textiles, which can be used for wearable systems. Wu et al. electrospun nonwoven mats of PZT fibers for energy harvesting, ${ }^{9}$ while Zheng et al. utilized a nanocomposite electrospun fiber to yield a wearable device. ${ }^{10}$

An alternative material to piezoceramics are piezoelectric polymers such as polyvinylidene fluoride (PVDF), which is a highly durable and environmentally stable polymer well suited for the manufacture of piezoelectric yarns. The process of extracting electrical energy from a piezoelectric material relies on the material's voltage coefficient, which is larger in many polymers than ceramics due to their low dielectric constant allowing an increase in electric field. Hadimani et al. developed a process to simultaneously spin and pole continuous PVDF fibers; ${ }^{11}$ they subsequently demonstrated the integration of the fibers into a three-layer knit in which the core transverse fibers were piezoelectric and the outer layers were conductive gold-coated nylon. ${ }^{12}$ This architecture leads to a fully integrated energy-harvesting textile and electrode system that provides strong energy-harvesting capability. Nanofiber poly(vinylidenefluoride-cotrifluoroethylene) (PVDF-TrFE) co-block polymers have also been demonstrated as a piezoelectric textile. ${ }^{13}$ The PVDF-TrFE textile is mechanically robust and produces $40 \mathrm{nA}$ and $1.5 \mathrm{~V}$ under bending. The addition of a graphene oxide (GO) layer between the PVDF-TrFE film and the bottom electrode has been reported to increase voltage and power output. ${ }^{14}$ This is due, in part, to the increased Young's 
modulus of the GO film, which increases the energy coupled to the PVDF-TrFE film.

\section{Piezoelectric films}

An alternative to using piezoelectric yarns is to use a standard fabric and then deposit a piezoelectric layer on top. Elkjaer et al. developed a piezoelectric paint that can be applied to any fabric via pad or screen printing. ${ }^{15}$ The piezopaint has a relatively high $d_{33}$ of $45 \mathrm{pC} / \mathrm{N}$ with a lead-free variant producing $25 \mathrm{pC} / \mathrm{N}$; these are both suitable for potential energy-harvesting applications.

Almusallam et al. developed a similar printable piezoelectric polymer by combining PZT powder with a flexible polymer and optimizing this process (Figure 2). ${ }^{16}$ This material was printed directly onto the fabric using screen printing, producing a freestanding $d_{33}$ value of $98 \mathrm{pC} / \mathrm{N}$. The electromechanical coupling of a piezoelectric paint or nanocomposite can be further enhanced through the use of acicular fillers such as nanowires. Zhou et al. demonstrated $7 \times$ greater energy harvesting from polymers with $\mathrm{PZT}^{17}$ and $5 \mathrm{Ba}(\mathrm{Zr} 0.2 \mathrm{Ti0}$.8)O3$0.5(\mathrm{Ba} 0.7 \mathrm{Ca} 0.3) \mathrm{TiO} 3(\mathrm{BZT}-\mathrm{BCT})^{18}$ nanowires embedded in the polymer over equiaxial fillers.

PVDF-TrFE is also available in printable form from Solvay, branded as Solvene electroactive polymers. ${ }^{19}$ Being a printable polymer ink, this material is in principle compatible with textiles, but are in practice difficult to screen print, and the $d_{33}$ value is low at $-25 \mathrm{pC} / \mathrm{N}$.

\section{Materials for thermoelectric energy harvesting}

Thermoelectric generators collect energy from a temperature difference due to the Seebeck effect. ${ }^{20}$ They are often made of semiconductor materials and work by thermally promoting electrons. The Seebeck effect is defined in Equation 1, where $S$ is the Seebeck coefficient in $\mathrm{VK}^{-1}, v$ is the potential produced by the thermal gradient in Volts, and $\Delta T$ is the temperature difference in degrees Kelvin, $\mathrm{K}$.

$$
S=\frac{v}{\Delta T}
$$


However, $S$ is just one aspect of a thermoelectric material, and a more complete picture of the relative merits of a material is given by the thermoelectric figure of merit $Z T$ represented by Equation 2:

$$
Z T=\frac{s^{2} \sigma T}{\kappa},
$$

where $\sigma$ is the electrical conductivity in $\mathrm{Sm}^{-1}, \kappa$ is the thermal conductivity in $\mathrm{Wm}^{-1} K^{-1}, T$ is the absolute temperature of the system in $K$. This demonstrates that improving a thermoelectric material requires an increase in the Seebeck coefficient or electrical conductivity, or a reduction in the thermal conductivity. $S^{2} \sigma$ is often referred to as the power factor as this shows the amount of energy that can be output in $\mathrm{Wm}^{-1} K^{-1}$.

The choice of materials for wearable thermoelectric devices can be split into inorganic- and organic-based devices. Inorganic devices are predominantly based on bismuth telluride $\left(\mathrm{Bi}_{2} \mathrm{Te}_{3}\right)$ for $p$-type and antimony telluride $\left(\mathrm{Sb}_{2} \mathrm{Te}_{3}\right)$ for $n$-type. ${ }^{21}$ Organic thermoelectric devices are less common due to poor performing materials and limited availability of $n$-type polymers. However, those that have been demonstrated use the conducting polymer poly(3,4-ethylenedioxythiophene) (PEDOT) with various dopants (typically polystyrene sulphonate [PSS]). ${ }^{22}$

Flexible thermoelectric energy harvesters are covered in more detail in another article within this issue. This article focuses on textile implementations. An early example of textile-based wearable thermoelectric devices used commercially available rigid ceramic-based thermoelectric generators (TEGs). These TEGs were simply stitched to clothing and were able to produce power outputs of $0.9 \mathrm{~mW}$ from a TEG area of $168 \mathrm{~cm}^{2}$ during sedentary activity. ${ }^{23}$ This approach was taken a step further by using smaller TEGs and integrating them into clothing along with a heatsink that produced a $30 \%$ increase in power output. $^{24}$

Since these early examples, the focus has moved toward flexible devices often fabricated by printing. ${ }^{25}$ One example used inorganic thermoelectric materials mixed with a polymer binder to form a screen-printable paste. ${ }^{26}$ The ratio of the ingredients is important to achieve a balance between flexibility and performance. When using an epichlorohydrin polyglycol-based epoxy binder, it 
was found that for $\mathrm{Bi}_{1.8} \mathrm{Te}_{3.2}$ and $\mathrm{Sb}_{2} \mathrm{Te}_{3}, 86 \mathrm{wt} \%$ inorganic material to binder was optimal for screen printing. A challenge for these materials is that high curing temperatures $\left(>250^{\circ} \mathrm{C}\right)$ were required to reduce material resistivity, which is not compatible with most textiles. The printed thermoelectric materials were shown to have room-temperature $Z T$ values of 0.135 and 0.095 for $\mathrm{Bi}_{1.8} \mathrm{Te}_{3.2}$ and $\mathrm{Sb}_{2} \mathrm{Te}_{3}$, respectively, which are close to the values of bulk materials and therefore very promising.

The current state of the art for organic thermoelectric materials is PEDOT:PSS ${ }^{27}$, which was shown to have a a room-temperature ZT of 0.42 , a considerable improvement on the 0.025 value achieved using commercially available solutions. This figure of merit was achieved by optimizing the ratio of PEDOT to PSS and using dimethyl sulfoxide to improve electrical conductivity. The advantage of using polymer-based materials is their low processing temperature of $\sim 100^{\circ} \mathrm{C}$, allowing them to be used on common fabrics.

Thermoelectric yarns have also been obtained using evaporated thin-film nickel and silver thermocouples on flexible fibers. ${ }^{28}$ The materials were alternated along the length of the fiber, producing 10-mm-long thermocouples. This material combination yielded a Seebeck coefficient of $19.6 \pm 0.6 \mu \mathrm{V} \mathrm{K}$, and each thermocouple produced $1 \mu \mathrm{W}$ from $100^{\circ} \mathrm{C}$ applied to the hot junction and with the cold junction at ambient.

\section{Photovoltaic films on textiles}

Fabric solar cells have great potential for powering wearable devices. However, the constraints imposed by the textile on the processes and materials that can be used result in the most significant challenge among the energy harvesting techniques considered in this review. Existing solar cells on fabrics use conventional rigid silicon or plastic solar cells that are attached (stitched or glued) onto the fabric as a functional patch. ${ }^{29}$ This approach makes the fabric inflexible and alters the feel of the textile dramatically, and the fabric itself has no added functionality. A new generation of flexible dye sensitized solar cells (DSSCs) and 
organic solar cells (OSCs) offers the potential for integrating the solar cells onto the fabric itself.

Bedeloglu et al. reported fabric OSCs by combining evaporation and spin coating. ${ }^{30}$ This work used a nonwoven polypropylene textile tape as the substrate, which is not representative of typical woven fabrics, and achieved a good power conversion efficiency of $0.2 \%$. Lee et al. fabricated OSCs on a flexible polyethylene terephthalate (PET)/ indium tin oxide substrate, which was then attached to a conductive fabric that acted as the bottom electrode. ${ }^{31}$ This approach does not add functionality to the textile itself and uses evaporation processes for some of the films. Recent research has investigated spray coating to deposit OSCs on a woven polyester cotton textile, as shown in Figure 3. The OSC is entirely solution processed, fabricated at low temperature $\left(<150^{\circ} \mathrm{C}\right)$, and uses a poly $(3$ hexylthiophene) (P3HT): indene-C60 bisadduct (ICBA)formulation, manufactured by Plextronics, for the active layer. Spray coating provides a repeatable and reliable process capable of depositing thin films onto the textile interface layer. Areas of the fabric where no films are required can be easily masked. Early work demonstrated a $0.02 \%$ power conversion efficiency (PCE), ${ }^{32}$ but a more recent version has achieved a PCE of $1.23 \%$, the highest reported for a solution-processed OSC on fabrics reported to date.

Other research has explored fabricating a functional organic photovoltaic fiber that can then be woven into a textile. ${ }^{33}$ The fabrication of quality functional organic thin films on the surface of a fiber is challenging. One example of successfully thermally evaporated thin films on polyimide coated silica fibers demonstrated a maximum efficiency of $0.5 \% .{ }^{34}$ Lee et al. used a double-twisted structure based on a conducting polymer and a soluble fullerene derivative photoactive layer deposited on a $100-\mu \mathrm{m}$-diameter stainless-steel wire. ${ }^{35}$ This arrangement demonstrated an efficiency of 3.7\%, although the inevitable partial shading and varying angle of incident light detrimentally affected the fiber PV output.

DSSCs are thin-film photoelectrochemical cells based on a semiconductor formed between a photosensitized anode and an electrolyte. There are several 
approaches reported in the literature to realize DSSC textiles, with many based on the production of single photovoltaic yarns subsequently woven into a textile. The yarns demonstrate a high efficiency of more than $7 \%$, but physical wear is a concern with deterioration apparent after 20 stretch cycles. ${ }^{36}$ Similar yarns have been woven together to form a DSSC that demonstrated an efficiency of $1.3 \%{ }^{37}$ Connecting a number of crossed yarn solar cells and weaving these into a textile remains a challenge for practical applications. The compatibility of DSSC with roll-to-roll processes such as printing and the inherently flexible materials make printed cells on textiles a potential solution. Printed low-temperature processed DSSCs on woven polyester cotton textiles have been demonstrated with an efficiency of $2.78 \%{ }^{38}$ However, these use a conventional liquid electrolyte, which requires resolving leakage, evaporation, and long-term stability issues in practical applications. Solid-state electrolytes are a potential way forward.

In recent years, perovskite solar cells (PSCs) have demonstrated rapid improvements in PCE up to a reported value of $22.1 \%{ }^{39}$ Most perovskite materials require high-temperature annealing at $450-500^{\circ} \mathrm{C}$, which is too high for direct processing on textiles. However, there are several reports of flexible PSCs with low-temperature techniques such as atomic layer deposition or thermal evaporation on PET and polyethylene naphthalate polymer substrates with the highest reported PCE of $14.4 \%{ }^{40}$ and $12.2 \%,{ }^{41}$ respectively. Flexible PSCs have also been achieved by printing using roll-to-roll slot-die printing and spray coating $t$ with an efficiency of $11.96 \% .{ }^{42}$ The processes used to realize flexible PSCs could be applied to textile substrates, but this has yet to be demonstrated. Qiu and Li et al. have also reported yarn-based flexible PSCs, both of which achieved a PCE of $3 \%{ }^{43,44}$

\section{Conclusions}

Numerous energy-harvesting textiles have been demonstrated that can successfully convert ambient energy into electrical energy for powering wearable and autonomous systems. Textiles are a challenging application for materials, limiting processing temperatures to typically $150^{\circ} \mathrm{C}$. Active films and yarns should be as compliant as possible to minimize any change in the feel of the 
textile. Active yarns woven into the textile must survive the weaving/knitting/embroidery process, and the textile manufacturing process can limit their location in the textile. Once incorporated into the textile, however, the yarn approach will maintain textile properties such as breathability. Film-based approaches enable the textile to be functionalized, and this can be done immediately after textile fabrication or, for example, after garment assembly. Printed films can be deposited in any pattern, but films will typically reduce breathability. There are remaining research challenges around the performance, mechanical properties, and practicality of the materials. However, textiles are found in numerous applications and industries, not just clothing, and suitable energy-harvesting materials developed for energy harvesting will have many uses.

\section{References}

1. K. Yang, R. Torah, Y. Wei, S. Beeby, J. Tudor, Text. Res. J. 83, 2023 (2013).

2. S.R. Anton, H.A. Sodano, Smart. Mater. Struct. 16, R1 (2007).

3. W. Liu, X. Ren. Phys. Rev. Lett. 103, 257602 (2009).

4. Y. Lin, H. Sodano, Adv. Funct. Mater. 19, 592 (2009).

5. Y. Lin, J.W. Shaffer, H.A. Sodano, Smart Mater. Struct. 19, 124004 (2010).

6. C. Bowland, Z. Zhou, H.A. Sodano, Adv. Funct. Mater. 24, 6303 (2014).

7. Y. Qin, X. Wang, Z.L. Wang, Nature 451, 809 (2008).

8. M.H. Malakooti, B.A. Patterson, H.-S. Hwang, H.A. Sodano, Energy Environ. Sci. 9, 634 (2016).

9. W. Wu, S. Bai, M. Yuan, Y. Qin, Z.L. Wang, T. Jing, ACS Nano 6, 6231 (2012).

10. W. Zeng, X.M. Tao, S. Chen, S. Shang, H.L.W. Chan, S.H. Choy, Energy Environ. Sci. 6, 2631 (2013).

11. R.L. Hadimani, D.V. Bayramol, N. Sion, T. Shah, L. Qian, S. Shi, E. Siores, Smart Mater. Struct. 22, 075017 (2013).

12. N. Soin, T.H. Shah, S.C. Anand, J. Geng, W. Pornwannachai, P. Mandal, D. Reid, S. Sharma, R.L. Hadimani, D.V. Bayramol, E. Siores, Energy Environ. Sci. 7, 1670 (2014). 
13. L. Persano, C. Dagdeviren, Y. Su, Y. Zhang, S. Girardo, D. Pisignano, Y. Huang, J.A. Rogers, Nat. Commun. 4, 1633 (2013).

14. V. Bhavanasi, V. Kumar, K. Parida, J. Wang, P.S. Lee, ACS Appl. Mater. Interfaces 8, 521 (2016).

15. K. Elkjaer, K. Astafiev, E. Ringgaard, T. Zawada, Proc. Ann. Conf.

Prognostics Health Mgmt. Soc. New Orleans, USA, 14-17 Oct., p. 372 (2013)

16. A. Almusallam, Z. Luo, A. Komolafe, K. Yang, A. Robinson, R. Torah, S. Beeby, Nano Energy 33, 146 (2017).

17. Z. Zhou, H. Tang, H.A. Sodano, Adv. Mater. 26, 7547 (2014).

18. Z. Zhou, C.C. Bowland, M. Malakooti, H. Tang, H.A. Sodano, Nanoscale 8, 5098 (2016).

19. Solvay, "solvene EAP for Printed Organic Electronics," available at http://www.solvay.com/en/binaries/Solvene_EAP_for_Printed_Electronics_EN220711.pdf (accessed October 24, 2017).

20. H.J. Goldsmid, Introduction to Thermoelectricity (Springer-Verlag Berlin Heidelberg, 2010).

21. H. Alam, S. Ramakrishna, Nano Energy 2, 190 (2013).

22. L.M. Cowen, J. Atoyo, M.J. Carnie, D. Baran, B.C. Schroeder, ECS J. Solid State Sci. Technol. 6, N3080 (2017).

23. V. Leonov, C. Van Hoof, R.J. Vullers, in Proc. Sixth Int. Workshop Wearable and Implantable Body Sensor Networks (IEEE, Berkeley, CA, 2009), p. 195.

24. V. Leonov, IEEE Sens. J. 13, 2284 (2013).

25. M. Orrill, S. LeBlanc, J. Appl. Polym. Sci. 134, 44256 (2017).

26. Z. Cao, M.J. Tudor, R.N. Torah, S.P. Beeby, IEEE Trans. Electron Devices 63, 4024 (2016).

27. G.H. Kim, L. Shao, K. Zhang, K.P. Pipe, Nat. Mater. 12, 719 (2013).

28. A. Yadav, K. Pipe, M. Shtein, J. Power Sources 175, 909 (2008).

29. M.B. Schubert, J.H. Werner, Mater. Today 9, 42 (2006).

30. A. Bedeloglu, P. Jimenez, A. Demir, Y. Bozkurt, W.K. Maser, N.S. Sariciftci, J. Text. Inst. 102, 857 (2011).

31. S. Lee, Y. Lee, J. Park, D. Choi, Nano Energy 9, 88 (2014). 
32. S. Arumugam, Y. Li, S. Senthilarasu, R. Torah, A.L. Kanibolotsky, A.R. Inigo, P.J. Skabara, S.P. Beeby, J. Mater. Chem. A 4, 5561 (2016).

33. D. Zou, Z. Lv, X. Cai, S. Hou, Nano Energy 1, 273 (2012).

34. B. O’Connor, K.P. Pipe, M. Shtein, Appl. Phys. Lett. 92, 193306 (2008).

35. M.R. Lee, R.D. Eckert, K. Forberich, G. Dennler, C.J. Brabec, R.A. Gaudiana, Science 324, 232 (2009).

36. Z. Yang, J. Deng, X. Sun, H. Li, H. Peng, Adv. Mater. 26, 2643 (2014).

37. N. Zhang, J. Chen, Y. Huang, W. Guo, J. Yang, J. Du, X. Fan, C. Tao, Adv. Mater. 28, 263 (2016).

38. J. Liu, Y. Li, S. Arumugam, J. Tudor, S. Beeby, Mater. Today Proc. $1^{\text {st }}$ Int. Conf. on Adv. Energy Mater. \& $8^{\text {th }}$ on Adv. NanoMater., Guildford, UK (2016).

39. W.S. Yang, B.-W. Park, E.H. Jung, N.J. Jeon, Y.C. Kim, D.U. Lee, S.S. Shin, J. Seo, E.K. Kim, J.H. Noh, S.I. Seok, Science 356, 1376 (2017).

40. Y. Du, H. Cai, X. Bao, Z. Xing, Y. Wu, J. Xu, L. Huang, J. Ni, J. Li, J. Zhang, ACS Sustainable Chem. Eng., 6 (1), pp 1083-1090 (2018).

41. B.J. Kim, D.H. Kim, Y.-Y. Lee, H.-W. Shin, G.S. Han, J.S. Hong, K. Mahmood, T.K. Ahn, Y.-C. Joo, K.S. Hong, N.-G. Park, S. Lee, H.S. Jung, Energy Environ. Sci. 8, 916 (2015).

42. K. Hwang, Y.-S. Jung, Y.-J. Heo, F.H. Scholes, S.E. Watkins, J. Subbiah, D.J. Jones, D.-Y. Kim, D. Vak, Adv. Mater. 27, 1241 (2015).

43. L. Qiu, J. Deng, X. Lu, Z. Yang, H. Peng, Angew. Chem. Int. Ed. 53, 10425 (2014).

44. R. Li, X. Xiang, X. Tong, J. Zou, Q. Li, Adv. Mater. 27, 3831 (2015).

\section{Figure Captions}

Figure 1. SEM micrographs of Kevlar fabric with $\mathrm{ZnO}$ nanowires grown on the surface to enable energy harvesting; the left image shows the Kevlar fibers and the right image is magnified, highlighting the $\mathrm{ZnO}$ nanowires. ${ }^{8}$

Figure 2. Digital photograph of (a) Flexible lead zirconate titanate (PZT) composite film on Kapton and an SEM micrograph of (b) poly(dimethylsiloxane) nanocomposite with PZT nanowires dispersed in the film, the magnified section in (b) highlights the density of the piezoelectric layer. ${ }^{16}$

Figure 3. Device structure (a) of a fully solution-processed spray-coated fabric substrate organic solar cell (OSC) and a digital photograph (b) the plan view of 
fabric OSC. Note: NW, nanowire; PEDOT, poly(3,4-ethylenedioxythiophene); PSS, polystyrene sulfonate; P3HT, , poly(3-hexylthiophene); ICBA, , indene-C60 bisadduct; NP, nanoparticle; IF, interface and coated fabrics. ${ }^{32}$

Russel Torah is a Senior Research Fellow in the Department of Electronics and Computer Science at the University of Southampton, UK. He obtained his BEng degree in electronic engineering in 1999, his MSc degree in instrumentation and transducers in 2000, and his $\mathrm{PhD}$ degree in electronics in 2004, all from the University of Southampton. His research focuses on functional materials development and e-textiles applications. He is a co-founder (in 2011) of the Smart Fabric Inks company, which specializes in printed smart fabrics. He has authored or co-authored 82 publications. Torah can be reached by phone at +44 (0)23 8059 2600 or by email at rnt@soton.ac.uk.

Jake Lawrie-Ashton is a doctoral candidate in the Smart Electronic Materials and Systems Group in the Department of Electronics and Computer Science at the University of Southampton, UK. He obtained his BSc degree in chemistry from the University of Southampton in 2016, with an electrochemistry focus. His current research interests include printable organic thermoelectric materials and wearable thermoelectric devices. Lawrie-Ashton can be reached by phone at +44 (0)2380595362 or by email at jla1g13@soton.ac.uk.

Yi Li is a Research Fellow in the Department of Electronics and Computer Science at the University of Southampton, UK. He obtained his BEng degree in electronic engineering in 2008 from the University of Warwick, UK, and his MSc degree in microsystem technology in 2009 and his $\mathrm{PhD}$ degree in printed smart fabrics in 2013 from the University of Southampton. His current research focuses on printed smart fabric-based electronic devices. Li can be reached by phone at +44(0)2380592353 or by email at yi.li@soton.ac.uk. 
Sasikumar Arumugam is a Research Fellow in the Department of Electronics and Computer Science at the University of Southampton, UK. He received his BSc degree in 2005 and his MSc degree in 2007 in Chemistry from Bishop Heber College, India. He was awarded a Scottish Overseas Research Award Scheme in 2010. His completed his doctoral studies in the Department of Chemistry at the University of Strathclyde, UK. Since 2013, he has been working as a Research Fellow on printed textile solar cells in the Smart Electronics and Materials group in ECS, Southampton. Arumugam can be reached by phone at +44 (0)23 8059 9318 or by email at S.Arumugam@soton.ac.uk.

Henry A. Sodano is a professor in the Departments of Aerospace Engineering, Materials Science and Engineering, and Macromolecular Science and Engineering at the University of Michigan. He received his BS degree in 2002, MS degree in 2003, and $\mathrm{PhD}$ degree in 2005 in mechanical engineering, all from Virginia Institute of Technology (Virginia Tech). His current research includes advanced materials, with a focus on composite materials, multifunctional materials, additive manufacturing, ceramics, and nanotechnology. His awards include the NSF CAREER Award in 2009, the American Society of Composites Young Researcher Award in 2012, the ASME Gary Anderson Award for Early Career Achievement in 2009, Virginia Tech's 2010 Outstanding Recent Alumni Award, and Arizona State University's 2009 Faculty Achievement Award in Research Excellence. He was inducted into Virginia Tech's Academy of Engineering Excellence in 2010. He is a Fellow of ASME and an Associate Fellow of AIAA. Sodano can be reached by phone at (734) 763-6728 or by email at hsodano@umich.edu.

Stephen P. Beeby has been a Professor of electronic systems and devices with the Department of Electronics and Computer Science, University of Southampton, UK since 2011. His received his PhD degree from the University of Southampton. His research for over 20 years has focused on printed active materials, sensors, and energy harvesting. He was awarded a EPSRC Fellowship to investigate 
screen-printed active materials for energy harvesting on fabrics. He leads the UK's Energy Harvesting Network and is a co-founder of Perpetuum Ltd. He is a Fellow of the IET and a member of the IEEE. He has more than 250 publications, eight patents, and an h-index of 42. Beeby can be reached by phone at +44 (0)23 80596663 or by email at spb@soton.ac.uk. 